Document downloaded from:

http://hdl.handle.net/10251/103605

This paper must be cited as:

García Sanjuan, F.; Jaén Martínez, FJ.; Nácher-Soler, VE. (2017). Tangibot: A tangiblemediated robot to support cognitive games for ageing people A usability study. Pervasive and Mobile Computing. 34:91-105. doi:10.1016/j.pmcj.2016.08.007

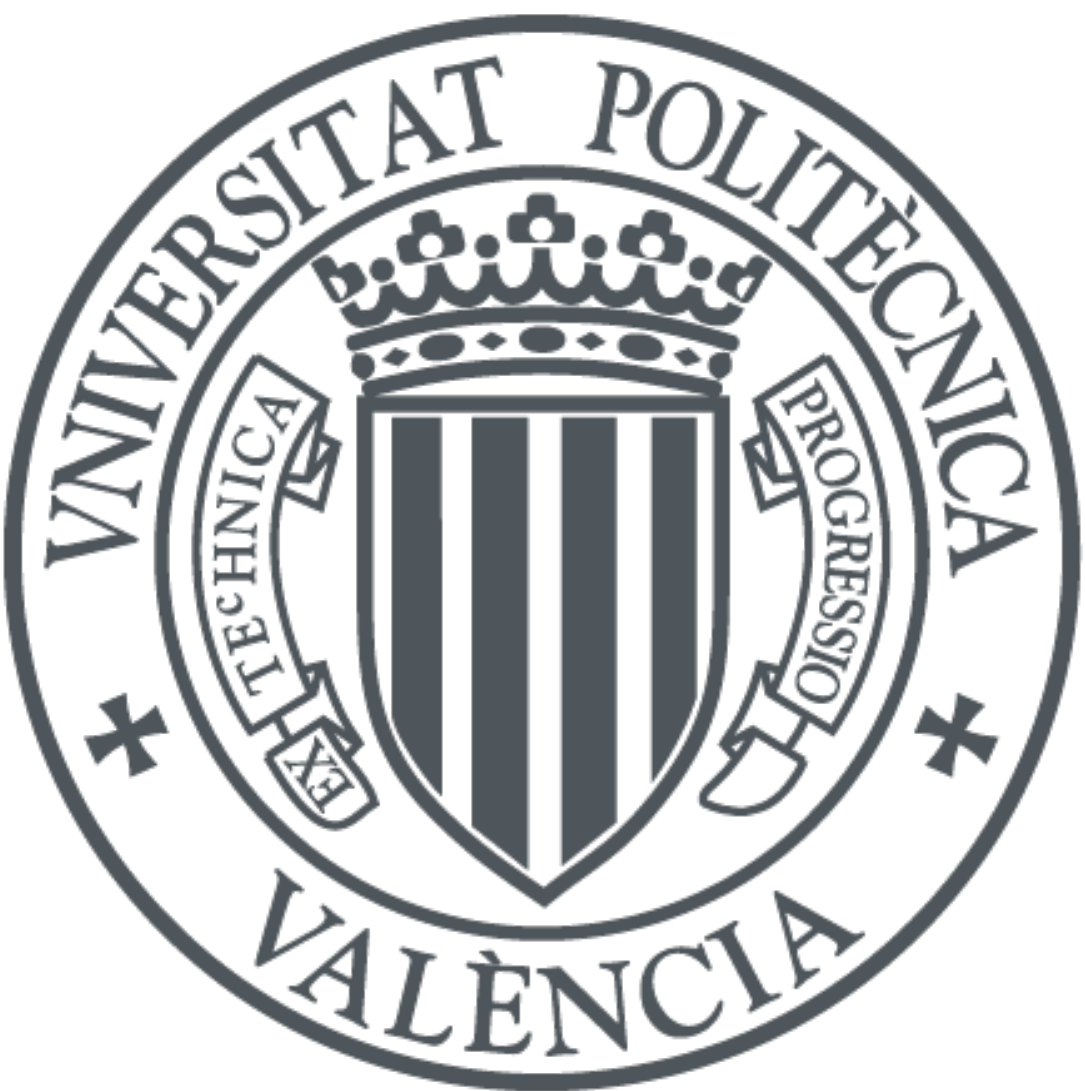

The final publication is available at

http://doi.org/10.1016/j.pmcj.2016.08.007

Copyright Elsevier

Additional Information 


\title{
Tangibot: A Tangible-Mediated Robot to Support Cognitive Games for Ageing People - A Usability Study
}

Fernando Garcia-Sanjuan, Javier Jaen, Vicente Nacher

\{fegarcia, fjaen, vnacher\}@dsic.upv.es

ISSI Group, Department of Computer Systems and Computation (DSIC)

Universitat Politècnica de València

46022 Valencia (Spain)

Phone: +34963873569

\begin{abstract}
The ever increasing elderly population requires a revision of technology to make it usable and meaningful for them. Most applications take into account their reduced physical and cognitive abilities in order to provide assistive services, but this paper focuses on building technology to improve these capacities through cognitive games. We present Tangibot, a tangible-mediated robot aimed at enabling more intuitive and appealing interactions. A usability study conducted on subjects at three different levels of cognitive impairment (none, mild, and severe) reveals that it is usable and engaging for users with no or mild cognitive impairment, and even though it is less usable for persons with severe impairment, it triggers positive emotional reactions among them, which makes it promising for their use in therapeutic activities.
\end{abstract}

\section{Keywords}

Gerontechnology, cognitive games, Tangible User Interfaces (TUI), robots, usability

\section{Introduction}

The number of ageing people in the European Union is increasing rapidly. According to Eurostat, the EU's elderly population is expected to rise from $17.9 \%$ in 2012 to $28.1 \%$ by the year 2050 due to the increase in average life expectancy and the continuous decline in birth rates [1]. This growth will require adapting existing technological services and creating new ones for this group of people [2]. 
The idea of ageing people and technology being incompatible is simply a cliché, as has already been shown in the literature. It is not true that the elderly have neither the capacity nor the will to learn and use new technologies. They do have the ability, although not necessarily the necessity [3]. It would appear that technological devices are normally designed for young people, and neither their purpose nor their interfaces appeal to the elderly. In fact, a study conducted by Fisk et al. [4] concluded that more than half the problems of this group with technology were associated with usability issues. In particular, the design of input/output devices and user interfaces is critical, as they interact with the user's perceptual and sensorial systems, which, at a certain age undergo changes that may have a negative impact on usability $[4,5]$. These changes include the loss of visual and acoustic capacities, touch- and movement-related issues (arthritis, trembling hands, mobility problems, etc.), and reduced cognitive capacities [6].

Traditionally, the most common way of interacting with computers were by mouse and keyboard, but these present severe usability issues that can cause the elderly to be reluctant to engage with technology [7]. Direct contact via touch interfaces has been shown to be a more suitable alternative for ageing users, since these interfaces present lower cognitive loads and spatial demands, and many efforts are being made of late to make this type of input device more intuitive [8]. Torres [7] proposed using graspable interfaces, which are typically referred to as Tangible User Interfaces (TUIs) [9]. These have already been used successfully in cognitive training activities [10] and offer spatial mapping, input/output unification, and support trial-and-error actions that can exploit innate spatial and tactile abilities, making these interfaces more natural and intuitive.

The present work presents a TUI prototype in the form of a mobile robot controlled by physical paddles aiming at creating games for the elderly to train their cognitive abilities (see Figure 1). The proposal consists of a generic and versatile technological device that allows both the elderly and the game designers (therapists) to easily create a range of activities. It also enables natural interactions through tangible manipulations and has the potential to foster socialization and the training of cognitive abilities that can improve the elders' quality of life. Specifically, the contributions of this paper are threefold. First, the design of said platform, called Tangibot; second, a usability study to assess whether the device can be used, and to which extent, by elderly users with different degrees of cognitive impairment; and finally, a discussion about the cognitive capacities that could be trained with Tangibot plus some examples of cognitive games that could be developed. 
The rest of the paper is structured as follows: Section 2 describes related work on technology for the elderly. Section 3 presents Tangibot's component parts. Section 4 describes a usability study of the technological platform, which is a first step before being able to build cognitive games for the elderly. Section 5 contains a description of the cognitive abilities that could benefit from training with Tangibot and some examples of cognitive games. Finally, Section 6 contains our conclusions and some ideas for future work.

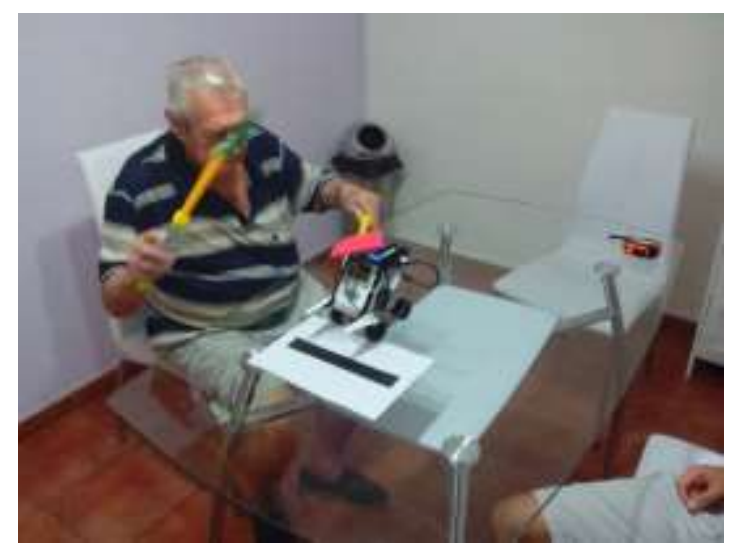

Figure 1. A user interacting with the platform

\section{Related work}

Many research studies have proposed methods of monitoring elderly people living on their own via ubiquitous devices (ambient or wearable) with minimum impact on their daily activities (e.g., [11-14]). Some focus on tracking the elderly outdoors, such as Lin et al. [14], who propose a method of detecting when a person gets disoriented when walking on the street, thus being able to provide real-time assistance. Others opt for tracking the elderly in their homes. Zhan et al. [12], for instance, present a device in the form of reading glasses to classify everyday activities based on what they are looking at and head movements. This system allows people to be tracked by their caregivers and warns them in case of any danger. In a similar fashion, Barsocchi et al. [13] present an indoor location mechanism that is able to detect deviations from normal behavior.

Other proposals that can be found in the literature also offer technology as a service to the elderly, but in the form of tools to help deal with age-related problems in respect of physical and cognitive capacities. Some of them, in the form of assistive robots or mobile applications (e.g., [15-18]), offer services to improve the quality of life of the elderly and enhance their independence. However, they are often described as aids rather than therapeutic devices to reduce the negative impact of their declining 
capacities. They are usually designed as personal devices, omitting socialization, even though ageing people seem to assign a high value to socializing and they even report being against technology when it replaces face-to-face interactions [19]. In terms of socialization, efforts have been made to use robots (called assistive social robots [20]) to maintain social relations with ageing users. However, they are not intended to foster human-to-human socialization. In fact, some authors have expressed their concerns that these technologies may actually increase social isolation [21]. As a counterexample, Nostalgia [22] is a TUI in the form of a textile runner and a radio which enables users to listen to $20^{\text {th }}$ Century music or to old news, which triggers discussion and socialization among the elderly.

In addition to robots, other groups propose the use of digital games (a.k.a., cognitive games) to stimulate declining cognitive abilities and foster socialization (e.g., $[5,23,24])$. In this regard, play represents an advantageous way to engage elderly users both cognitively and socially [6]. There are many references in the literature that stress the benefits of videogames for the elderly. They have been proved to decrease reaction times $[25,26]$ and improve quality of life, self-confidence, and cognitive skills (the latter two showing a positive correlation) [7]. Whitcomb [23] observed that when ageing people played a series of videogames their social interaction improved as did their perceptual-motor capacities (eye-hand coordination, dexterity, fine motor ability, and a reduction of the reaction time). Although the author did not explicitly study how videogames affected cognitive capacities, the study detected a positive effect of videogames on information processing, reading, comprehension, and memory.

Interaction design for videogames for the elderly is a critical dimension to be considered. Whitcomb [23] enumerates several characteristics that make a videogame unsuitable for them, such as small-sized objects, rapid movements or reactions required, and inappropriate sound. In terms of interaction mechanisms, this study focuses on computer games with interactions mainly transmitted through mouse and keyboard. However, as mentioned in Section 1, other interaction mechanisms may be advantageous for the elderly. In this respect, authors such as Jung et al. [27] explored other input/output devices, e.g., a Wii stick in a game to enhance general wellbeing (physical activity, self-esteem, affection, and level of solitude). However, in our opinion, this type of interaction should be considered with caution when the elderly are involved, as it has been known to produce physical injuries such as tendinitis (or Wiiitis, as it has been called) [28]. Alternatively, Chiang et al. [29] report elderly users significantly improving their visual performance skills through Kinect games. Others have taken advantage of the increasing popularity 
of handheld devices, which can be moved around and do not require the user to stay in the same position to play (i.e., in front of the television or the computer). MemoryLane [5], although not exactly a game, fosters reminiscence through a PDA application to create "memory stories" with pictures. Vasconcelos et al. present CogniPlay [24], a gaming platform running on tablets which includes several games to stimulate cognitive abilities, such as matching pairs to enhance short-term memory and social interaction through competition. De La Guía et al. [30,31] also explore cognitive games for the elderly using smartphones and tangibles to increase the engagement of ageing users. However, the consideration of small displays may entail, on the one hand, visualization problems for the elderly. On the other hand, they are usually used as private ( single user) devices, which is clearly a step in the wrong direction when collaboration needs to be fostered.

Other works also aim to stimulate either cognitive abilities and/or socialization by taking advantage of the natural and intuitive manipulations that physical (tangible) elements can offer, so that users can focus more on the activity than on controlling the platform. E-CoRe [32] and IntouchFun [33] are two examples of tangible-mediated cognitive games that run on tabletops. The latter also enables remote socialization between the elderly and their families, but this socialization does not take place between several users in the same place. The Virtual Fishing game [34] enables co-located experiences in which several users sit next to one another to "fish" in a digital tabletop using a tangible fishing rod, but no cognitive capacities are stimulated. The previous three works rely on tabletops, which nowadays present an elevated economic cost that prevents them from being implanted in many retirement homes. Age Invaders [35] is an intergenerational game platform that makes use of RFID-enhanced shoes to interact with an interactive floor, and it aims to foster social and physical interaction between elders and their (grand)children, however, it is not suitable for people with limited mobility (e.g., those in a wheelchair), who cannot move around the floor. CurBall [36], on the other hand, is another intergenerational game in which ageing users do not need to move. In this work, the players manipulate a virtual ball by physically manipulating a tangible proxy of it. However, this approach also relies on watching a digital element move on a screen, which, as stated above, could cause visualization issues.

Our approach aims to provide both a tangible element to control (a mobile robot) and a tangible way of interacting with it (some physical paddles). Making the interaction usable will enable us to devise games that help the elderly improve their declining cognitive capacities. Ultimately, we would like to build a 
technological platform that is both appealing to users and that can be used by multiple players at the same time in the same place in order to foster social relationships.

\section{Technological platform}

The prototype presented in this work consists of two major components: a mobile robot (see Figure 2) and a set of paddles as tangible mechanisms to communicate with it (Figure 3). Each paddle consists of an extendable stick (maximum length $45 \mathrm{~cm}$ ), which would enable users with reduced mobility to give commands to the robot from a distance by bringing them close to it, without relying on indirect input mechanisms. Also, the use of multiple paddles would allow therapists to design multiuser activities to foster socialization between the participants, in which the different paddles would be distributed among them. To make the paddles more appealing and to visually encode the associated command, they have a distinctive design in EVA foam attached to it via Velcro ${ }^{\circledR}$ strips, which facilitates their replacement. Each figure contains an RFID tag and represents a movement command to the robot, i.e. shift forward (green triangle, as in Figure 3-left), stop (red square), turn right (yellow circle), and turn left (blue circle). The use of RFID tags is motivated by their low cost and versatility, since each tag encodes an ID that can be mapped to a specific command for the robot and be easily embedded in the EVA-foam shapes. The robot was constructed using the Lego ${ }^{\mathrm{TM}}$ Mindstorms ${ }^{\circledR}$ Ev3 platform, as it is an affordable commercial product and it facilitates rapid prototyping of multiple versions. It communicates by Bluetooth with an external mobile phone connected to an RFID reader. The phone is able to process the RFID tags in the paddles and sends the corresponding movement control commands to the robot. It has an ornamental fork on the front in order to help the users distinguish between the front and back.



Figure 2. Details of the mobile robot 




Figure 3. Extendable robot control paddles. From left to right: shift forward, stop, turn left, turn right

Figure 4 depicts the communication process between the robot's components when a user gives a certain movement command to the robot. When (s)he brings the paddle close enough to the RFID reader, the RFID tag is read by the phone. The Android application running on the mobile device decodes the id and sends the corresponding command via Bluetooth to the Robot's CPU (i.e., "Forward", "Stop", "Left", and "Right"). Then, depending on the command, the CPU sends the appropriate message to the wheel's motors. When the command is "Forward" or "Stop", both motors move or stop together. When the robot is commanded to turn, a motor is rotated -50 degrees (backwards) and the other is rotated 50 degrees (forward).

The program running on the robot is coded using the visual programming metaphor of the Lego ${ }^{\mathrm{TM}} \mathrm{Ev} 3$ platform (see Figure 5) which enables the rapid prototyping of different types of movement behaviors.

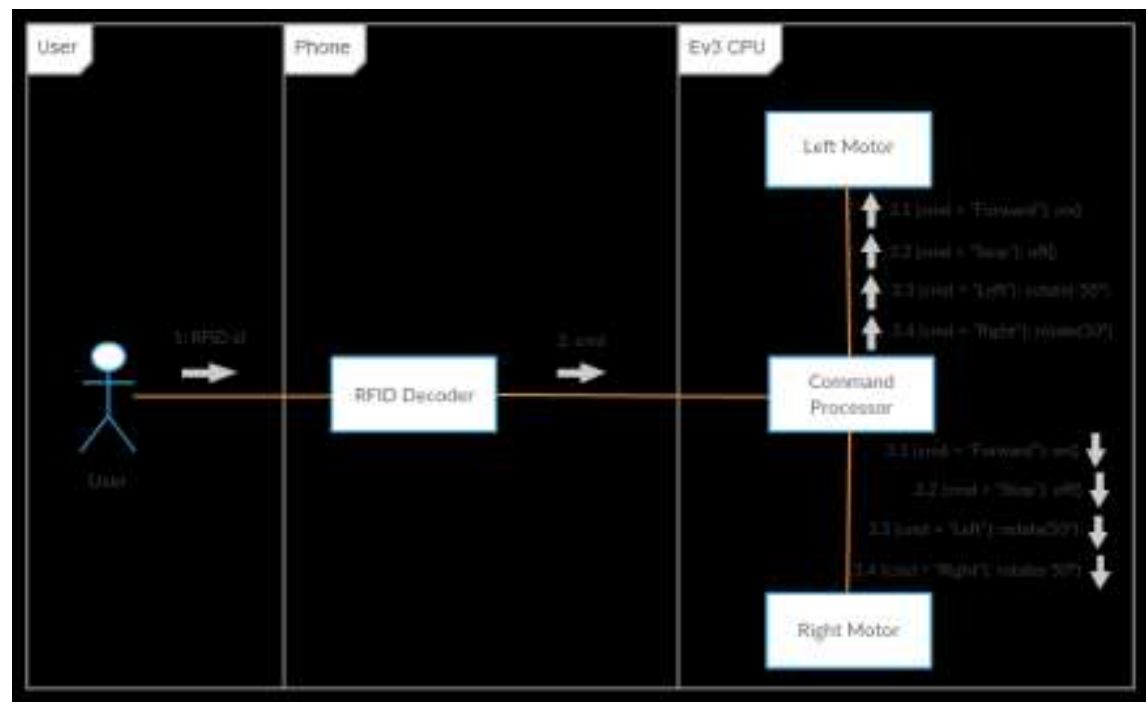

Figure 4. Messages sent between the robot's components when a movement command $(\mathrm{cmd})$ is sent 


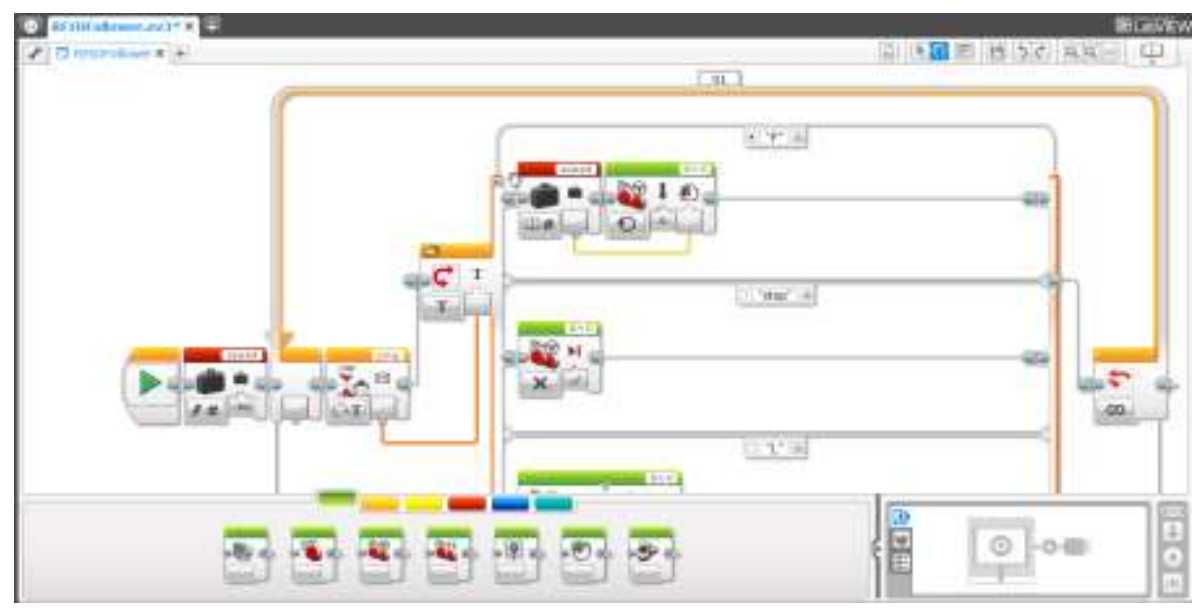

Figure 5. Mindstorms ${ }^{\mathrm{TM}}$ Ev3 visual programming environment

\section{Usability evaluation}

Before starting on the design of complex activities or cognitive games for the elderly on this platform, it is necessary to know whether this robot and its TUI are usable and appealing to these people, taking into account the cognitive issues they might have. This section describes an in-lab study conducted with real users with different degrees of cognitive impairment and that consisted basically of controlling the robot's position and orientation with the paddles in order to make it match the corresponding position and orientation of a target.

\subsection{Apparatus and participants}

Besides the robot and the four paddles, a sheet of paper with a thick black line drawn in the middle was used as a target. The experiment was conducted on a 130x60 cm rectangular table (see Figure 1).

Forty-six residents of three different retirement homes were asked to participate in this study. Whereas four of them refused even to try and two more quit after the first contact with the platform, the remaining forty agreed to participate until the end of the experiment. Their ages ranged from 57 to 95 years $(\mathrm{M}=$ 81.33, $\mathrm{SD}=8.48)$ and thirty-two were females.

The therapists in charge of the subjects classified them into three groups according to their level of cognitive impairment: none, mild, and severe, regardless of their age. Those with no cognitive impairment were totally independent and capable of reasoning normally. Those diagnosed with mild cognitive issues presented early symptoms of dementia, but were still capable of maintaining a conversation and performing most of their routines. Finally, the ones selected with severe cognitive 
impairment were unable to maintain a meaningful conversation and their short-term memory was highly defective, although they were still capable of understanding basic instructions. Sixteen subjects had no cognitive impairment, twelve had mild, and twelve others were severely impaired.

\subsection{Tasks and procedure}

Each user was asked to perform three different tasks: one to control the orientation of the robot, another to shift it from one location to another, and a third as the combination of the first two. Each task was repeated four times. The orientation and shifting tasks required handling only two paddles at a time, and in order to avoid carryover and order effects, they were presented alternatively first and second to each user. The third task was always the combination of the previous ones because it was intrinsically more complex, not only because it required more operations but because it required the users to manage four paddles at a time.

For the orientation task, the target line was situated on an imaginary circumference (with radius $=30 \mathrm{~cm}$ ) around the robot and pointing towards it at a random angle of between $0^{\circ}$ and $360^{\circ}$. The goal was to rotate the robot until it faced the target, as shown in Figure 6-left. In the shifting task, the target was situated in front of the robot at a random distance of between 0 and $100 \mathrm{~cm}$, as if it were a finish line (see Figure 6right). In this case, the robot always followed a straight horizontal trajectory and the subjects were instructed to stop it when the robot's fork reached the target's line. For the last task, which combined orientation and shifting, both the robot and the target were placed at random positions on the table and the subjects first had to make the robot face the target and then make it move towards it, as in the previous activities. In all three tasks, after each repetition, the orientation and position parameters were changed. Also, since the concepts of right and left change depending on whether the robot is facing towards or away from the user, after each repetition of a task involving rotation, the robot's initial orientation was alternatively changed from facing the subject to looking away from him. 


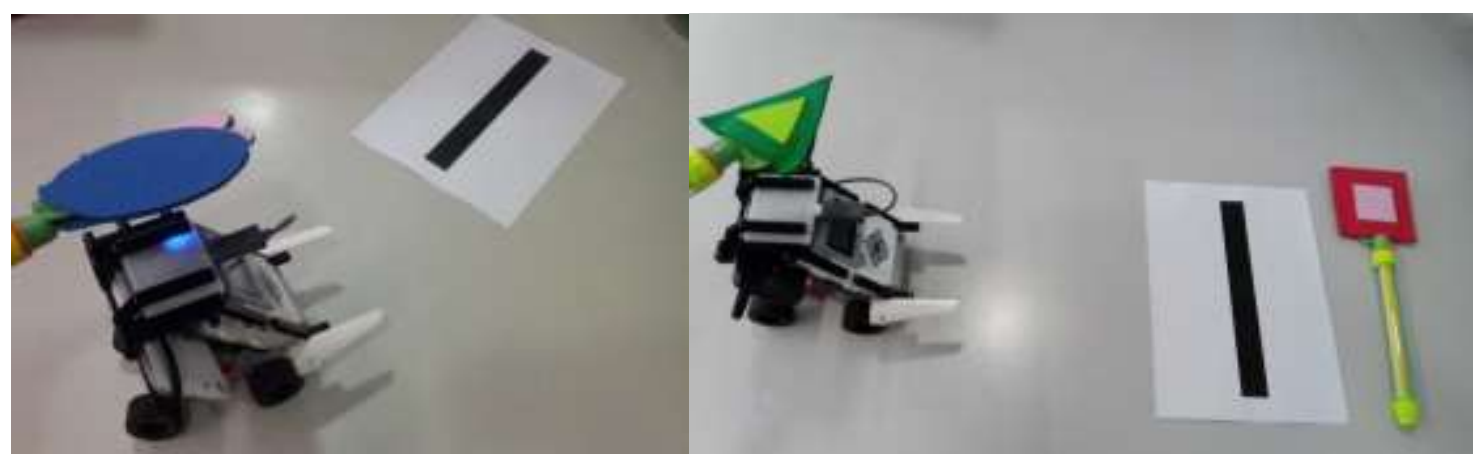

Figure 6. Setup for the orientation task (left) and the shifting task (right)

Each subject performed the three tasks individually seated at the center of the table (see Figure 1). Before each task, the subjects were explained the interactions they would have to perform to complete the task, and were given some time to train with the platform until they felt confident enough to begin. For those with communication problems, the supervisor decided when they were ready.

\subsection{Design}

For each task (orientation, shifting, and all combined), the effect of the level of cognitive impairment (none, mild, or severe) was evaluated. The following variables were measured:

- Proportion of repetitions completed: For each user, this variable describes how many times (s)he was able to bring the robot to its target orientation and/or position, depending on the manipulation being evaluated.

- Time: For those repetitions of tasks that were successfully completed, this variable measures the time the users spent performing interactions until its completion.

- Unnecessary actions: If a given repetition of a task was completed, this variable measures the difference between the number of actual actions performed (i.e., turn right, turn left, shift, stop) and the optimal number of actions the task would require, namely, 1 action for orientation tasks (turn left or right continuously till the robot faces the target line), 2 actions for shifting tasks (one to start the movement and another to stop), and 3 actions for the final task in which all the paddles were available (one action to turn, one to start the movement, and a third to stop the robot at its destination).

- Robot shifting precision errors: For those tasks when the shifting of the robot was available, this variable indicates the distance between the robot fork and the center of the black target line. 
- Failed actions: This variable measures the percentage of actions the participants tried to perform and failed by not bringing the paddle close enough to the RFID reader.

Since the administration of questionnaires to obtain subjective feedback was discarded due to the inability of some users to understand the questions, their gestures, reactions, and comments were recorded during the course of the different tasks in order to identify two sets of variables: their impressions of the platform and their different behaviors during the activity.

\subsection{Results}

\subsubsection{Proportion of repetitions completed}

Figure 7 shows the proportion of repetitions completed by the users of the different groups for each task. Although the users with no or mild impairment were able to complete most orientation and shifting tasks, this proportion was reduced when they were given all four paddles. An ANOVA $(\alpha=0.05)$ showed a statistically significant effect of the level of impairment on the proportion of repetitions completed, both for the orientation $\left(\mathrm{F}_{2,37}=12.548 ; p<0.001\right)$ and shifting $\left(\mathrm{F}_{2,37}=6.766 ; p=0.003\right)$ tasks. Post-hoc Bonferroni pairwise comparisons showed subjects with severe cognitive impairment failed to complete significantly more repetitions than those with a lesser degree of impairment $(p<0.01)$, but no significant differences were found in this respect between mild cognitive impairment or none $(p>0.85)$. Since the hypothesis of homoscedasticity did not hold for the task combining orientation and shifting $\left(\mathrm{F}_{2,37}=5.671\right.$; $p=0.007)$, a Kruskal-Wallis $H$ test $(\alpha=0.05)$ revealed no significant differences between the different levels of impairment on the dependent variable for this task $\left(\chi^{2}(2)=4.958 ; p=0.084\right)$.

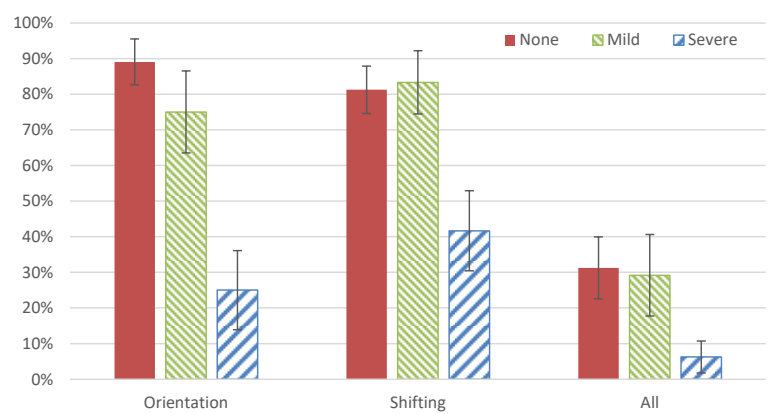

Figure 7. Proportion of repetitions completed by subjects with no (none), mild, or severe cognitive impairment (grouped by task) 


\subsubsection{Time}

As depicted in Figure 8, users completed the shifting task faster ( 10s on average), followed by the orientation one ( $\sim 20$ s on average), and the combination of these two was the most time-consuming, in general. An ANOVA $(\alpha=0.05)$ revealed a significant effect of the cognitive impairment level on the orientation task $\left(\mathrm{F}_{2,102}=4.146 ; p=0.019\right)$. Post-hoc Bonferroni pairwise comparisons showed that the participants with severe cognitive impairment performed significantly slower $(p<0.03)$ than the rest, although no significant differences were found between those without or with a mild degree $(p=1.0)$. No differences were found either among groups for shifting tasks $\left(\mathrm{F}_{2,109}=0.273 ; p=0.762\right)$. With respect to the task in which all four commands were allowed, the hypothesis of homoscedasticity did not hold $\left(\mathrm{F}_{2,34}\right.$ $=3.368 ; p=0.046)$. Therefore, a Kruskal-Wallis H test $(\alpha=0.05)$ was conducted, which revealed a significant effect of the level of impairment $\left(\chi^{2}(2)=8.479 ; p=0.014\right)$. Post-hoc pairwise comparisons using a Mann-Whitney $U$ test revealed that the only two groups presenting a significant difference were those with no and mild cognitive impairment $(U=64.0 ; p=0.008)$, the former outperforming the latter. The ones with severe cognitive impairment presented similar mean completion times to the ones with a mild level.

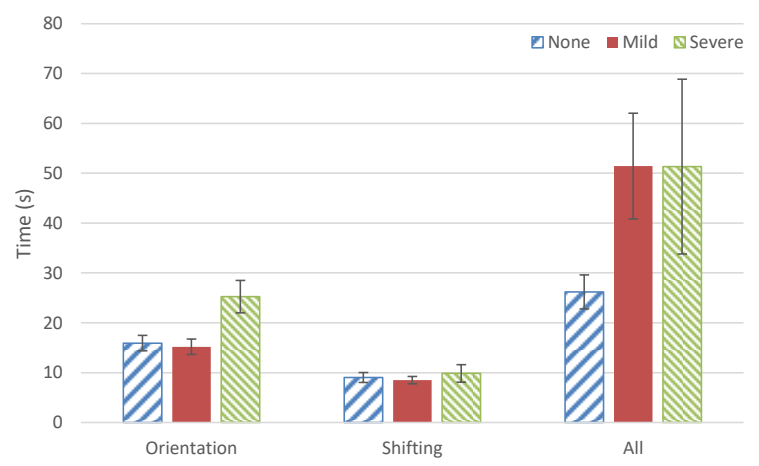

Figure 8. Time to complete a repetition of a task by subjects with no (none), mild, or severe cognitive impairment (grouped by task)

\subsubsection{Unnecessary actions}

As depicted in Figure 9, the users performed, on average, less than one extra action to complete the shifting tasks. A few more additional actions were performed when orientation was allowed (in the other two tasks), but normally no more than 3 or 4 . A Kruskal-Wallis H test $(\alpha=0.05)$ showed no significant effect of the impairment level on the dependent variable for both orientation and shifting tasks $(p>0.05)$. However, it did reveal a significant effect for the tasks using all four paddles $\left(\chi^{2}(2)=7.409 ; p=0.025\right)$. Post-hoc Mann-Whitney U tests revealed the subjects without cognitive issues performed significantly 
fewer unnecessary actions than the ones with mild cognitive impairment, but no differences were found between these two groups and the one with severe impairment, probably because of the high dispersion of the data, as depicted in Figure 9, which indicates that the participants with severe cognitive issues presented either very few unnecessary actions or many $(\sim 5)$.

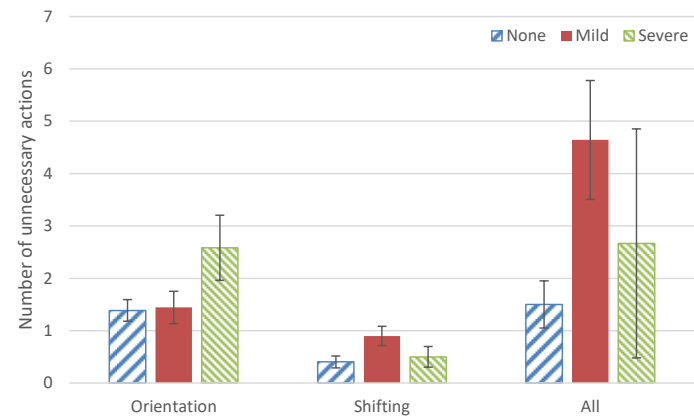

Figure 9. Number of unnecessary actions when completing a repetition of a task by subjects with no (none), mild, or severe cognitive impairment (grouped by task)

\subsubsection{Robot shifting precision errors}

As depicted in Figure 10, the users were able to stop the robot relatively close to the target (normally at less than $10 \mathrm{~cm})$ in both tasks in which shifting was available. Although an ANOVA $(\alpha=0.05)$ did not find any significant differences between levels of cognitive impairment for the task with all four paddles $\left(\mathrm{F}_{2,34}=0.335 ; p=0.718\right)$, it did reveal a significant effect of this factor on the shifting task $\left(\mathrm{F}_{2,109}=4.382\right.$ $p=0.015)$. Post-hoc Bonferroni pairwise comparisons revealed that the group with severe impairment was significantly less precise $(p<0.05)$ when trying to stop the robot at a specific point.



Figure 10. Shifting precision errors measured as the distance to the target when completing a repetition of a task by subjects with no (none), mild, or severe cognitive impairment (grouped by task)

\subsubsection{Failed actions}

Figure 11 depicts the proportion of times the participants tried to give a command to the robot and failed because they did not bring the paddle close enough to the robot's RFID reader. A Kruskal-Wallis H test 
$(\alpha=0.05)$ was performed on this variable and showed a significant effect of the subjects' level of cognitive impairment $\left(\chi^{2}(2)=29.4 ; p<0.001\right)$. Post-hoc Mann-Whitney U tests revealed the users with severe cognitive issues making significantly more failed actions $(p<0.001)$ than the rest, yet it was, on average, $10.33 \%(\mathrm{SD}=0.184)$. No significant differences were found between the participants with no or mild cognitive impairment $(\mathrm{U}=10856.0 ; p=0.958)$, whose actions were almost always successful.



Figure 11. Percentage of actions that were not successful at giving a command to the robot (grouped by level of cognitive impairment)

\subsubsection{Observational findings}

The users' impressions with regard to the platform were analyzed, and are summarized in Table 1 for each level of cognitive impairment. After completing the task, they were asked directly about whether they had liked the platform, and 32 out of $40(80 \%)$ answered affirmatively. However, the researchers noticed in some cases their answers did not seem sincere, maybe because they did not fully understand the question and/or they were trying to be polite. Instead, their spontaneous comments and reactions were observed during the session and subsequently analyzed. In this respect, 25 out of 40 users $(62.5 \%)$ showed clear manifestations of enjoyment, although these were more frequent in users as they presented lower cognitive impairment. They either laughed or expressed directly they were enjoying the activity. Concretely, of these 25 users, $20 \%$ of them found it entertaining (“It's very funny!”, "it's super-fun!”, "this is great!"), $36 \%$ did not want to stop playing ("I could spend all day playing with this little robot", “we have to stop already? Now that I was getting a taste for it...”), and $28 \%$ could not wait for the next repetition of the task to be set up and did not stop giving the robot commands. Besides, four users that were reluctant to use it at first because they thought they "could not do it right", ended up having fun with it (and two of them did not want to stop in the end). Furthermore, 13 out of 40 participants (32.5\%) found the robot "nice", "pretty", or "astonishing", and were the ones with mild cognitive impairment who expressed less comments about it. Nevertheless, 4 out of $40(10 \%)$ expressed clear impressions of 
contempt and irritation during the task. They found it "silly" and "useless". In this respect, one of them claimed: "What dumb things they do nowadays..." These expressions were more frequent among participants with severe cognitive impairment.

\begin{tabular}{|c|c|c|c|c|}
\hline & None (out of 16) & Mild (out of 12) & Severe (out of 12) & Total (out of 40) \\
\hline $\begin{array}{l}\text { Report liking the } \\
\text { platform }\end{array}$ & $15(93.75 \%)$ & $9(75 \%)$ & $8(66.67 \%)$ & $32(80 \%)$ \\
\hline $\begin{array}{l}\text { Manifest } \\
\text { enjoyment }\end{array}$ & $12(75 \%)$ & $7(58.33 \%)$ & $6(50 \%)$ & $25(62.5 \%)$ \\
\hline $\begin{array}{l}\text { Found it } \\
\text { entertaining }\end{array}$ & $2(12.5 \%)$ & $2(16.67 \%)$ & $1(8.33 \%)$ & $5(12.5 \%)$ \\
\hline $\begin{array}{l}\text { Do not want to } \\
\text { stop playing }\end{array}$ & $3(18.75 \%)$ & $2(16.67 \%)$ & $4(33.33 \%)$ & $9(22.5 \%)$ \\
\hline $\begin{array}{l}\text { Cannot wait for } \\
\text { the next } \\
\text { repetition }\end{array}$ & $4(25 \%)$ & $1(8.33 \%)$ & $2(16.67 \%)$ & $7(17.5 \%)$ \\
\hline $\begin{array}{l}\text { Praise the robot's } \\
\text { beauty }\end{array}$ & $7(43.75 \%)$ & $2(16.67 \%)$ & $4(33.33 \%)$ & $13(32.5 \%)$ \\
\hline Express contempt & 0 & $1(8.33 \%)$ & $3(25 \%)$ & $4(10 \%)$ \\
\hline
\end{tabular}

Table 1. Number (and proportion) of users with each level of cognitive impairment that expressed the specified impressions

Other observations made during the experiment concerned the behaviors that emerged during the interactions, and are summarized in Table 2 for each level of cognitive impairment. Many users (57.5\%), regardless of their level of cognitive impairment, needed help at some point to complete the tasks if they forgot what they were doing or which paddle represented which command. This help provided by the researchers or the therapists consisted of reminding them to bring the robot to match the target's position and/or orientation if they noted some distraction on the subject, and telling them what paddle entailed 
which movement in case the participant asked. In no case was the solution to the task revealed. It was also observed that 9 out of $40(22.5 \%)$ users usually started the interaction with the paddle they already had in their hands or whichever was closest, and only 7 (17.5\%) planned in advance which interactions to perform. Another remarkable behavior observed was that during the task with all four paddles, 6 users (15\%) discovered that they could stop the robot not only with the red-squared paddle, but also with those for turning, and then put aside the former, so that they would have to handle fewer paddles at once. It is worth noting that no user with severe cognitive impairment was able to plan in advance nor discovered the alternative way of stopping the robot. Some other minor findings were: 5 users $(12.5 \%)$ reinforced their manual commands with words by talking to the robot, and 4 participants (10\%), none of which had a mild cognitive impairment, cheated at some point by moving the robot with their hands or bringing the target to the robot. We consider they cheated and not simply were confused or did not understand the task because when they did they naughtily laughed about it.

\begin{tabular}{|c|c|c|c|c|}
\hline & None (out of 16) & Mild (out of 12) & Severe (out of 12) & Total (out of 40) \\
\hline $\begin{array}{l}\text { Need help at } \\
\text { some point }\end{array}$ & $10(62.5 \%)$ & $5(41.67 \%)$ & $8(66.67 \%)$ & $23(57.5 \%)$ \\
\hline $\begin{array}{l}\text { Start with the } \\
\text { closest paddle }\end{array}$ & $6(37.5 \%)$ & $1(8.33 \%)$ & $2(16.67 \%)$ & $9(22.5 \%)$ \\
\hline Plan in advance & $3(18.75 \%)$ & $4(33.33 \%)$ & 0 & $7(17.5 \%)$ \\
\hline $\begin{array}{l}\text { Discover many } \\
\text { ways to stop }\end{array}$ & $4(25 \%)$ & $2(16.67 \%)$ & 0 & $6(15 \%)$ \\
\hline Talk to the robot & $3(18.75 \%)$ & $1(8.33 \%)$ & $1(8.33 \%)$ & $5(12.5 \%)$ \\
\hline Cheat & $2(12.5 \%)$ & 0 & $2(16.67 \%)$ & $4(10 \%)$ \\
\hline
\end{tabular}

Table 2. Number (and proportion) of users with each level of cognitive impairment that showed the specified behaviors 


\subsection{Discussion}

The previous results indicate that Tangibot is usable by ageing people with none or mild cognitive impairments but is too demanding for those with severe cognitive impairments. In general, the participants with none or mild cognitive impairments were able to complete most of the exercises presented to them that required the use of two paddles at a time (i.e., the orientation and shifting tasks), and within a reasonable time. When handed the four paddles to make the robot both rotate and shift towards a target, the number of repetitions they were able to complete was considerably reduced (with mean success rates of $31.25 \%$ for the participants without impairment, and $29.17 \%$ for the ones with mild issues), and needed more time for those they did perform (mostly when they presented a mild cognitive impairment). This could be explained by this kind of task being cognitively more complex since they could not hold all four paddles in their hands at once and had to remember and manage more commands. As explained in Section 4.4.6, this is why $15 \%$ of the users discarded some paddles when they found they could achieve similar results with the other ones (i.e., the stop action was included as a pre-action in the paddles for turning right or left). These results suggest that, in order to design future cognitive games with this platform, a single user should handle at most two commands at once. Multi-user activities could be built in which two or more users had to collaborate to solve a problem by jointly controlling the robot (each one being in charge of one or two paddles). This way we could foster not only the development of cognitive abilities but also socialization among peers.

As Figure 12 depicts, when a user was unable to successfully complete a given repetition of a task, it was due to three main reasons: inactivity (i.e., remaining still not knowing what to do), aimlessness (i.e., interacting with the robot without a clear goal, making it move arbitrarily), and fall (i.e., not being able to stop the robot before falling off the table, where the researcher had to intervene and grab it). During the task where the users controlled the orientation of the robot only, the main reason of incompletion was aimlessness, since the robot could not fall off the table. However, the more cognitive impairment the subjects had, the more cases appeared when they remained inactive in front of the robot not knowing how to proceed with the interaction. For shifting tasks, the most common reason for not being able to complete a repetition was a fall, because they made it move and kept staring at it idly. Surprisingly, all the users presented the same reasons in the same proportions regardless of their level of impairment. During the final task in which they handled all the paddles, the three previous reasons occurred. As they had more sever cognitive impairment, the robot falling off the table gave way to aimlessness and inactivity. 


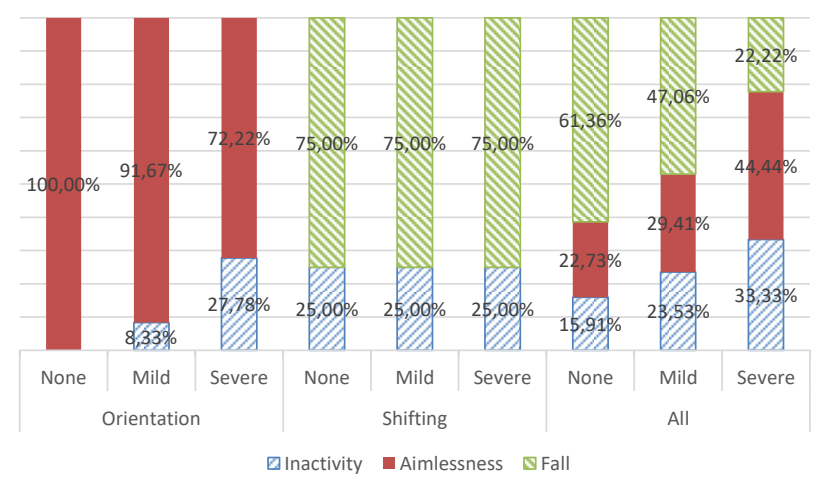

Figure 12. Reasons to not being able to complete a repetition of a task (grouped by task and level of cognitive impairment)

The subjects with none or mild cognitive issues also achieved a fair degree of precision in terms of a reduced number of unnecessary actions and a small distance between the robot and the target when making the former move towards the latter. With respect to the number of extra actions performed, however, the users performed a few more in the orientation than in the shifting task. This occurred because, in some cases, they confused directions, and did not understand why, using the same paddle, the robot sometimes turned to their right when it was looking at them and sometimes to their left when it was facing in the opposite direction. During the shifting task they were more accurate in this respect. This could be due to them finding the interaction more intuitive, since they were able to complete, on average, more than $80 \%$ of the repetitions. As happened with the time to complete the task, when they were handling all four paddles, the subjects with mild cognitive impairment performed significantly more unnecessary actions than those without impairment, probably because they needed more trials to fully understand the task. Nevertheless, in general terms, having a mild cognitive impairment seems not to be a hindrance to using this platform.

Elderly people with severe cognitive issues were significantly less successful in completing the tasks than the ones with none or mild impairments. In the task where orientation and shifting were combined, however, the statistical analysis did not reveal a significant effect of the cognitive impairment level, although the users with a severe one presented lower mean values of the number of completed tasks (6.25\% versus $31.25 \%$ and $29.17 \%)$. Nonetheless, in the orientation and shifting tasks, where they only had to handle two paddles at once, the analysis of several variables indicates the worse performance of these users. For example, they spent significantly more time and performed more unnecessary actions on average than the other two groups, rotating the robot in the orientation task, but no differences were found in the shifting task. This is probably because the former required them to be more precise in order to 
consider the task completed (i.e., they had to make the robot look at the target), which entailed correcting their actions if they missed the target, whereas the shifting task was considered successful even if the robot stopped without precision after the "finish line" was crossed. This reason is supported by the significantly higher shifting precision errors in the shifting task with respect to the other groups of users. Moreover, the subjects with a severe impairment presented significantly more failed actions because they could not fully understand and/or remember where they needed to put the paddle for the robot's RFID reader to detect it.

To sum up, from a quantitative perspective, it can be concluded that Tangibot would probably be more suited to subjects with none or mild cognitive impairments. According to their personal preferences, the less cognitive impairment they presented the more they seemed to like the platform as well (both by expressing more enjoyment and less contempt). However, regardless of the cognitive issues, $62.5 \%$ of the users showed clear manifestations of enjoyment when interacting with the robot, which indicates that the platform could be a useful tool for triggering positive emotional reactions even in the case of persons with severe cognitive impairments (50\% of them showed positive manifestations). Even in cases in which some users were reluctant to engage in the activity because they were afraid they could not perform well, they finally ended up having a good time. During subsequent discussions with the therapists, they stated that many users (mostly the ones with none or mild impairments) talked among themselves about the experience with Tangibot, and some of them, after completing the task, went running to their families to tell them how they had been handling something so complex as a robot and how well they had performed. Many elderly participants told us their grandchildren would "love" such a platform as well. For this reason we believe that intergenerational activities with Tangibot could be worth exploring in the future.

\subsection{Threats to validity}

There are some limitations in our study. On the one hand, the small proportion of repetitions completed by the users with severe cognitive impairment in the task where all four paddles were available $(6.25 \%$ on average) has resulted in high standard deviation of the data and, as a result, a lack of statistical power, which has complicated the comparison between those participants with severe issues and the other two groups of users for some dependent variables: time and unnecessary actions.

On the other hand, as pointed out by Glisky [37], it is important to note that deficits in perception (visual, acoustic, etc.) have a significant impact on cognition, therefore they could also have an impact on the 
usability of Tangibot. In our study we have not taken into account these perceptual impairments and have only classified the users with respect to the level of cognitive impairment, a complementary study would be necessary to check the possible effects of perceptual deficits on the usability of our platform.

\section{Designing cognitive games for ageing people with Tangibot}

\subsubsection{Age-related reduction of cognitive abilities}

According to Glisky [37], there are three basic cognitive functions that diminish with age and could be trained:

- Attention: It spans across virtually all other cognitive domains, except when the task at hand is automatized. There are two types of attention that are affected by age: selective and divided. Whereas the former consists on being able to focus on some stimuli while filtering out other irrelevant ones, divided attention requires processing multiple sources of information or performing multiple tasks at the same time.

- Working memory: It is probably the main source of age-related deficits that has an impact in many other cognitive domains such as language, problem solving, and decision making. It involves the active manipulation of information that is currently being maintained in the task at hand.

- Long-term memory: It requires the retrieval of information that is no longer present or being maintained in an active state. It is also composed of several subtypes, but the ones specifically affected by normal ageing are the following: episodic memory (i.e., being able to remember personally experienced events in a specific place at a particular time), and prospective memory (i.e., remembering to do things in the future) when no reminders in the environment are available (e.g., remembering when to take a specific medication).

In addition to those basic functions, Glisky also distinguishes other, higher-level, cognitive capacities which become affected by ageing. Some of them, e.g., language or decision making, are not reduced per se on the elderly, but they are affected by working memory loss. On the other hand, she identifies executive control as a high-level cognitive function that is indeed a "primary contributor to cognitive decline with age". Executive control is "a multi-component construct that consists of a range of different processes that are involved in the planning, organization, coordination, implementation, and evaluation of many of our routine activities". 
Finally, there are other cognitive functions that according to Glisky are not necessarily affected by ageing such as sustained attention (i.e., being able to maintain concentration over an extended period of time), semantic memory (i.e., remembering factual facts, words, and concepts), autobiographical memory (i.e., memories about oneself), procedural memory (i.e., the knowledge of skills such as riding a bicycle), and implicit memory (which refers to "a change in behavior that occurs as a result of prior experience, although one has no conscious or explicit recollection of that prior experience"). Although some of these (procedural and implicit memory) probably could not be trained using our platform, the remaining ones could also be trained by designing cognitive games with Tangibot.

\subsubsection{Examples of cognitive games}

Several studies (e.g., [38-40]) as well as some therapists from the retirement homes where the experiment described in Section 4 took place remarked the importance of training cognitive abilities in the elderly in order to prevent or slow down their decline over time. Given the good acceptance of Tangibot among the participants, we consider it a motivating element for older people to engage in cognitive games that help them train such capacities. Four generic samples of such games are explained below, classified by the main cognitive capacity they are designed to train. All of them have the same basic idea: control the robot's movements with the paddles in order to make it either follow a path or reach a destination.

\subsubsection{Selective attention}

This game would consist on the adaptation of the "visual search" activity, a classic to train selective attention [37]. The therapists would place a target image on a table, and around it, they would arrange a bunch of other images acting as distractors. Selective attention is trained in this activity since users would need to filter out the distractors and locate the target image. What they should do is make the robot move and make it stop on top of the target image. The complexity of the task could be increased by augmenting the number of distractors or by making them more similar to the target. According to Rogers [40], the selective attention depends on the familiarity of the user with the presented objects. Taking this into account, in a low complexity level the images to memorize could be easily recognizable by the participants, and the game could be made more challenging by introducing pictures of objects they are not familiar with. 


\subsubsection{Working memory}

Working memory is trained intrinsically with Tangibot since users have to remember which paddle entails which command to the robot. However, a game example to train working memory could be to arrange several images on a table for the user to memorize where each one is (as shown in Figure 13). Next, the therapist would turn them upside down, and then ask the user to bring the robot to one of them. The complexity could be increased by adding more images for the user to remember or by making him/her bring the robot to many targets.

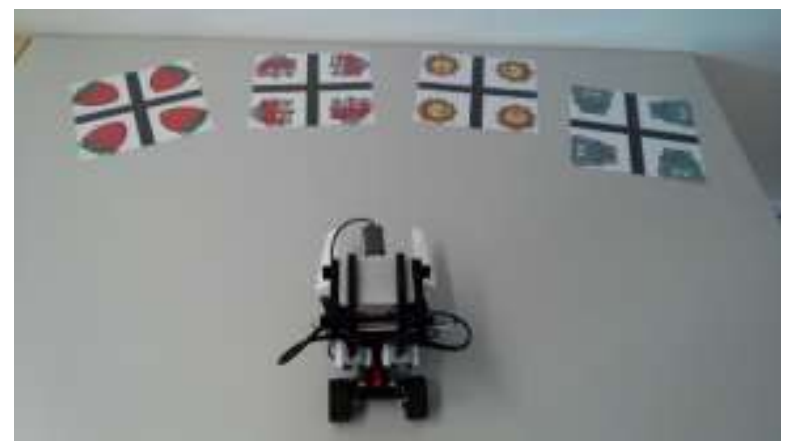

Figure 13. Example of a cognitive game to stimulate working memory with Tangibot

Another game to train working memory could consist of the therapist drawing multiple paths on the table, then making the robot follow a specific path, and, finally, having the players make the robot repeat that trajectory. The complexity in this case could be increased by adding more possible paths for the user to choose from or by making them longer.

\subsubsection{Episodic memory}

In a similar way to Carthy et al. [5], a storytelling activity could be designed in order to foster reminiscence. In our context, several pictures of the user's life could be placed in a chronological order on the table. Then, the user would have to bring the robot to one of them and, when on top of it, (s)he would have to talk about what they remember of the moment that photo represents. The therapist could increase the difficulty by presenting the images unsorted for the user to visit in the correct order. Also, this task could be used to train selective attention by adding some meaningless pictures, which the user would have to avoid.

\subsubsection{Prospective memory}

The therapist would arrange (unsorted) several images on the table from the user's daily routine, for example, representations of the meds (s)he needs to take during the day. Then, in a similar way as the 
previous game to foster episodic memory, the older participant should visit all images in the correct order. The images could represent any succession of tasks the users need to conduct, and the complexity of the game can also be increased by adding more stimuli, either meaningful or not (if selective attention wants to be trained).

\subsubsection{Stimulating other capacities}

Besides the specific cognitive abilities the four previous games are designed to train, since they make use of Tangibot other capacities can be stimulated. For example, executive control is exercised because controlling the robot requires certain planning and coordination of actions. Also, divided attention is trained since users need to focus on controlling the robot at the same time as they need to complete the game's task.

Another important aim of this platform is avoiding the dangers of social isolation that could provide a similar implementation where each user would hold a tablet or be in front of a computer screen. As suggested by the results of Section 4.4, each user should not be in control of more than two paddles at a time. Therefore, there should be devised collaborative scenarios where several people situate around the table and help one another find better solutions, and/or simply discuss the game and the situation themselves. Also, since some users felt proud of their performance (see Section 4.5), in our opinion, these activities using Tangibot could also foster self-confidence in older adults.

Not only could Tangibot be used to train cognitive abilities and foster socialization. In our opinion, it could also be used in the context of physical rehabilitation or exercising. The mechanism to control the robot with the paddles trains coarse motor skills. Fine motor skills could also be stimulated by making the robot rotate and move to a specific target with precision. For those users able to walk, a therapist could draw a path and the users would have to make the robot follow it by walking by its side.

\subsubsection{Putting it all together}

Tangibot could also be used to build more complex games that integrate the training of several cognitive and physical abilities. For example, we have already prototyped a collaborative game to foster creativity. In this case, the therapist places several images on the table. Then, the users need to build a path by putting some wooden tiles together that connects all the images in order to, finally, make the robot visit all the images by walking on top of the wooden path (see Figure 14). After having completed this task, 
they would be asked to repeat the process by finding different paths to solve the same problem, thereby promoting creativity. Besides, this game would also train coarse and fine motor skills (because the robot could not leave the wooden path), and it would also stimulate collaboration (since each user would be in charge of a paddle and all together should come up with a solution to the problem), executive control in the form of planning and coordination, divided attention (since they would be conducting several operations at the same time), working memory (not to repeat a path defined previously), and selective attention (because they would be encouraged to make the solutions as optimal as possible, hence requiring them to discard irrelevant paths). A preliminary test was made with real users, in which we found the users to effectively socialize, find different solutions, and have fun. These preliminary findings illustrate the versatility of Tangibot and make this specific activity a promising area for future work.

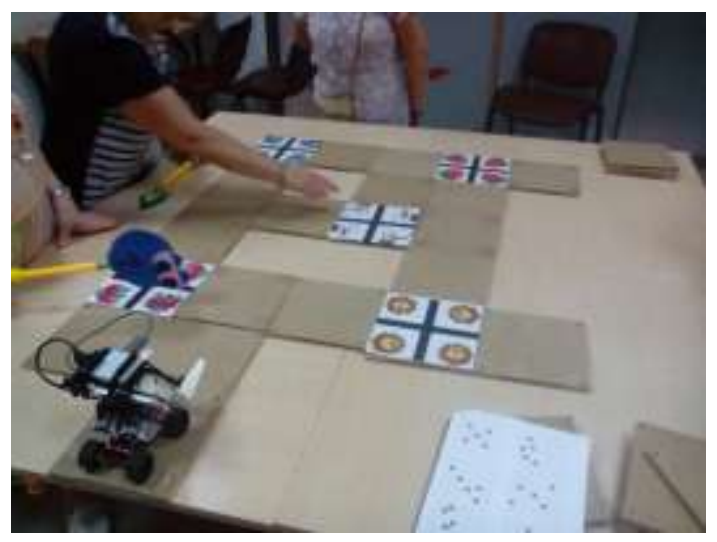

Figure 14. Four users playing a game to foster creativity with Tangibot

\section{Conclusions}

In this paper we present a prototype of Tangibot, a mobile robot mediated by four tangible paddles, which is a platform for constructing cognitive games for the elderly. The design would not only be able to foster human-to-human socialization but also the tangible capabilities would bring more natural and intuitive interactions that would appeal to ageing users. The platform is built of cost-effective materials, and its design allows for a quick setup and high versatility and scalability.

We conducted a study with 40 subjects and concluded that Tangibot is generally usable by older adults with none or mild cognitive impairments. The study also revealed that it may be too complex for those with severe cognitive issues. However, regardless of their cognitive impairments, the platform was found to be appealing to most participants, which, in our opinion, would make Tangibot a promising technological device that could serve as a motivating technological artifact in games to foster cognitive 
abilities. Our study also revealed that elderly players should only handle two paddles at most, which would enable the construction of multi-player games in which each player would be in charge of giving a movement command to the robot.

We have also provided several examples of games to train the cognitive capacities, and have also glimpsed the design of a more complex game aimed at stimulating many of these abilities, plus some physical ones and other higher-level capabilities such as creativity.

As future work, additional experiments would be conducted in order to study the positive effect of the platform on the already enumerated capacities meant to be stimulated. We will delve deeper into the usability of Tangibot by checking the possible effects of users' gender, specific cognitive impairments, and, as mentioned in Section 4.6, perceptual deficits. Also, in order to take advantage of the smartphone in the platform, we will consider augmenting the experience with Tangibot by providing visual and acoustic feedback to the users; for example, by providing visual and audio clues to help users with acoustic and visual issues, respectively. Additionally, we plan to exploit the capabilities of RFID technology by embedding tags in other common objects and study the impressions of ageing people towards interacting with the robot via those other elements. Finally, we will also explore the game described in Section 5.1.2.6 to foster creativity, and, since Tangibot has already been found usable for young children in another study [41], different scenarios will be examined in which the platform is used conjointly by elders, young adults, and children to foster intergenerational activities.

\section{Acknowledgements}

This work is supported by Spanish Ministry of Economy and Competitiveness and funded by the European Development Regional Fund (EDRF-FEDER) with Project TIN2014-60077-R. It is also supported by fellowship ACIF/2014/214 within the VALi+d program from Conselleria d'Educació,

Cultura i Esport (Generalitat Valenciana), and by fellowship FPU14/00136 within the FPU program from Spanish Ministry of Education, Culture, and Sport.

We would like to thank CEAM Cabanyal, Centro Sociosanitario Ntra. Sra. del Carmen, and Instituto Geriátrico Valenciano for their collaboration during the development of this study.

\section{References}

[1] Eurostat, Europe in figures - Eurostat yearbook: Population structure and ageing, (2014). 
http://ec.europa.eu/eurostat/statistics-explained/index.php/Population_structure_and_ageing (accessed July 27, 2015).

[2] F. Nunes, P.A. Silva, F. Abrantes, Human-computer interaction and the older adult: an example using user research and personas, in: Proc. 3rd Int. Conf. Pervasive Technol. Relat. to Assist. Environ., ACM, New York, NY, USA, 2010: pp. 49:1-49:8. doi:10.1145/1839294.1839353.

[3] J. Durick, T. Robertson, M. Brereton, F. Vetere, B. Nansen, Dispelling ageing myths in technology design, in: Proc. 25th Aust. Comput. Interact. Conf. Augment. Appl. Innov. Collab., ACM, New York, NY, USA, 2013: pp. 467-476. doi:10.1145/2541016.2541040.

[4] A.D. Fisk, W.A. Rogers, N. Charness, S.J. Czaja, J. Sharit, Designing for older adults: Principles and Creative Human Factors Approaches, CRC Press, 2004.

[5] S.M. Carthy, H. Sayers, P.M. Kevitt, M. McTear, MemoryLane: Reminiscence for Older Adults, in: Proc. First Int. Work. Reminisc. Syst., 2009: pp. 22-27.

[6] L. Gamberini, M. Alcaniz, G. Barresi, M. Fabregat, F. Ibanez, L. Prontu, Cognition, technology and games for the elderly: An introduction to ELDERGAMES Project., PsychNology J. 4 (2006) 285-308. http://www.psychnology.org/File/PNJ4(3)/PSYCHNOLOGY_JOURNAL_4_3_GAMBERINI.pd $\mathrm{f}($ accessed January 13, 2015).

[7] A.C.S. Torres, Cognitive effects of video games on old people, Int. J. Disabil. Hum. Dev. 10 (2011) 55-58. doi:10.1515/IJDHD.2011.003

[8] B. Loureiro, R. Rodrigues, Multi-touch as a Natural User Interface for elders: A survey, in: 6th Iber. Conf. Inf. Syst. Technol., IEEE, 2011: pp. 1-6.

[9] H. Ishii, B. Ullmer, Tangible bits: towards seamless interfaces between people, bits and atoms, in: Proc. SIGCHI Conf. Hum. Factors Comput. Syst., ACM Press, New York, New York, USA, 1997: pp. 234-241. doi:10.1145/258549.258715.

[10] E. Sharlin, Y. Itoh, B. Watson, Y. Kitamura, S. Sutphen, L. Liu, et al., Spatial tangible user interfaces for cognitive assessment and training, in: A.J. Ijspeert, M. Murata, N. Wakamiya (Eds.), Biol. Inspired Approaches to Adv. Inf. Technol., Springer Berlin Heidelberg, 2004: pp. 137-152. doi:10.1007/978-3-540-27835-1_11.

[11] K. Rieping, G. Englebienne, B. Kröse, Behavior analysis of elderly using topic models, Pervasive Mob. Comput. 15 (2014) 181-199. doi:10.1016/j.pmcj.2014.07.001. 
[12] K. Zhan, S. Faux, F. Ramos, Multi-scale Conditional Random Fields for first-person activity recognition on elders and disabled patients, Pervasive Mob. Comput. 16 (2015) 251-267. doi:10.1016/j.pmcj.2014.11.004.

[13] P. Barsocchi, M.G.C.A. Cimino, E. Ferro, A. Lazzeri, F. Palumbo, G. Vaglini, Monitoring elderly behavior via indoor position-based stigmergy, Pervasive Mob. Comput. (2015). doi:10.1016/j.pmcj.2015.04.003.

[14] Q. Lin, D. Zhang, K. Connelly, H. Ni, Z. Yu, X. Zhou, Disorientation detection by mining GPS trajectories for cognitively-impaired elders, Pervasive Mob. Comput. 19 (2015) 71-85. doi:10.1016/j.pmcj.2014.01.003.

[15] M. Montemerlo, J. Pineau, N. Roy, S. Thrun, V. Verma, Experiences with a mobile robotic guide for the elderly, in: Proc. 18th Natl. Conf. Artif. Intell., AAAI, Menlo Park, CA, USA, 2002: pp. 587-592. doi:10.1.1.141.6428.

[16] B. Otjacques, M. Krier, F. Feltz, D. Ferring, M. Hoffmann, Helping older people to manage their social activities at the retirement home, in: Proc. 23rd Br. HCI Gr. Annu. Conf. People Comput. Celebr. People Technol., British Computer Society, Swinton, UK, 2009: pp. 375-380. http://dl.acm.org/citation.cfm?id=1671059.

[17] J. Goodman, S. Brewster, P. Gray, Older people, mobile devices and navigation, in: Proc. HCI 2004, HCI Older Popul. Work., 2004: pp. 13-14.

[18] I. Shklovski, Y.-C. Chung, R. Adams, Robotic walker interface: designing for the elderly, in: Ext. Abstr. 2004 Conf. Hum. Factors Comput. Syst., ACM Press, New York, New York, USA, 2004: p. 1566. doi:10.1145/985921.986142.

[19] S. Eggermont, H. Vandebosch, S. Steyaert, Towards the desired future of the elderly and ICT: Policy recommendations based on a dialogue with senior citizens, Poiesis Prax. 4 (2006) 199217. doi:10.1007/s10202-005-0017-9.

[20] J. Broekens, M. Heerink, H. Rosendal, Assistive social robots in elderly care: a review, Gerontechnology. 8 (2009) 94-103. doi:10.4017/gt.2009.08.02.002.00.

[21] A. Sharkey, N. Sharkey, Granny and the robots: Ethical issues in robot care for the elderly, Ethics Inf. Technol. 14 (2012) 27-40. doi:10.1007/s10676-010-9234-6.

[22] M. Nilsson, S. Johansson, M. Håkansson, Nostalgia: An Evocative Tangible Interface for Elderly Users, in: CHI ’03 Ext. Abstr. Hum. Factors Comput. Syst., 2003: pp. 964-965. 
doi:10.1145/765891.766096.

[23] G.R. Whitcomb, Computer games for the elderly, in: Proc. Conf. Comput. Qual. Life, ACM, New York, NY, USA, 1990: pp. 112-115. doi:10.1145/97351.97401.

[24] A. Vasconcelos, P.A. Silva, J. Caseiro, F. Nunes, L.F. Teixeira, Designing tablet-based games for seniors: the example of CogniPlay, a cognitive gaming platform, in: Proc. 4th Int. Conf. Fun Games, ACM, New York, NY, USA, 2012: pp. 1-10. doi:10.1145/2367616.2367617.

[25] J.E. Clark, A.K. Lanphear, C.C. Riddick, The effects of videogame playing on the response selection processing of elderly adults, J. Gerontol. 42 (1987) 82-85. doi:10.1093/geronj/42.1.82.

[26] R.E. Dustman, R.Y. Emmerson, L.A. Steinhaus, D.E. Shearer, T.J. Dustman, The effects of videogame playing on neuropsychological performance of elderly individuals, J. Gerontol. 47 (1992) 168-171. doi:10.1093/geronj/47.3.P168.

[27] Y. Jung, K.J. Li, N.S. Janissa, W.L.C. Gladys, K.M. Lee, Games for a Better Life: Effects of Playing Wii Games on the Well-Being of Seniors in a Long-Term Care Facility, in: Proc. Sixth Australas. Conf. Interact. Entertain., ACM, New York, NY, USA, 2009: pp. 5:1-5:6. doi:10.1145/1746050.1746055.

[28] J. Bonis, Acute Wiiitis, N. Engl. J. Med. 353 (2007) 2431-2432. doi:10.1056/NEJMc070670.

[29] I.-T. Chiang, J.-C. Tsai, S.-T. Chen, Using Xbox 360 Kinect Games on Enhancing Visual Performance Skills on Institutionalized Older Adults with Wheelchairs, in: IEEE Fourth Int. Conf. Digit. Game Intell. Toy Enhanc. Learn., IEEE, 2012: pp. 263-267. doi:10.1109/DIGITEL.2012.69.

[30] E. de la Guía, M.D. Lozano, V.M.R. Penichet, Increasing Engagement in Elderly People through Tangible and Distributed User Interfaces, in: Proc. 8th Int. Conf. Pervasive Comput. Technol. Healthc., 2014: pp. 390-393. doi:10.4108/icst.pervasivehealth.2014.255361.

[31] E. de la Guia, M.D. Lozano, V.M. R. Penichet, Cognitive Rehabilitation Based on Collaborative and Tangible Computer Games, in: Proc. 7th Int. Conf. Pervasive Comput. Technol. Healthc., IEEE, 2013: pp. 389-392. doi:10.4108/icst.pervasivehealth.2013.252375.

[32] G.H. Kwon, L. Kim, S. Park, Development of a Cognitive Assessment Tool and Training Systems for Elderly Cognitive Impairment A case study of a successful development of a cognitive assessment tool and training systems for the elderly people in South Korea, in: Proc. 7th Int. Conf. Pervasive Comput. Technol. Healthc., 2013: pp. 7-9. 
doi:10.4108/icst.pervasivehealth.2013.252384.

[33] V. Meza-kubo, A.L. Morán, M. Rodríguez, IntouchFun, a Pervasive Collaborative System to Cope with Elder's Isolation and Cognitive Decline, in: Proc. 2010 ACM Conf. Ubiquitous Comput., ACM, 2010: pp. 1-4. http://www.createnet.org/ubint/ubihealth/papers/Meza_IntouchFun_final.pdf.

[34] H. Kim, Y. Roh, J.-I. Kim, An Immersive Motion Interface with Edutainment Contents for Elderly People, in: Motion in Games, Springer Berlin Heidelberg, Berlin, Heidelberg, 2008: pp. 154-165. doi:10.1007/978-3-540-89220-5_16.

[35] A.D. Cheok, Age Invaders: Entertainment for Elderly and Young, in: Art Technol. Entertain. Comput. Commun., Springer London, London, 2010: pp. 137-160. doi:10.1007/978-1-84996137-0_6.

[36] D. Kern, M. Stringer, G. Fitzpatrick, A. Schmidt, Curball - A prototype tangible game for intergenerational play, in: Proc. 15th IEEE Int. Work. Enabling Technol. Infrastruct. Collab. Enterp., IEEE, 2006: pp. 412-417. doi:10.1109/WETICE.2006.27.

[37] E.L. Glisky, Changes in Cognitive Function in Human Aging, in: D.R. Riddle (Ed.), Brain Aging Model. Methods, Mech., CRC Press, 2007: pp. 3-20. doi:10.1201/9781420005523.sec1.

[38] P.B. Baltes, D. Sowarka, R. Kliegl, Cognitive training research on fluid intelligence in old age: What can older adults achieve by themselves?, Psychol. Aging. 4 (1989) 217-221. doi:10.1037/0882-7974.4.2.217.

[39] G.E. Smith, P. Housen, K. Yaffe, R. Ruff, R.F. Kennison, H.W. Mahncke, et al., A Cognitive Training Program Based on Principles of Brain Plasticity: Results from the Improvement in Memory with Plasticity-based Adaptive Cognitive Training (IMPACT) Study, J. Am. Geriatr. Soc. 57 (2009) 594-603. doi:10.1111/j.1532-5415.2008.02167.x.

[40] W.A. Rogers, Attention and aging, in: D.C. Park, N. Schwarz (Eds.), Cogn. Aging A Prim., USA, Psychology Press, New York, NY, 2000: pp. 57-73.

[41] F. Garcia-Sanjuan, J. Jaen, V. Nacher, A. Catala, Design and Evaluation of a Tangible-Mediated Robot for Kindergarten Instruction, in: Proc. 12th Int. Conf. Adv. Comput. Entertain. Technol., ACM, 2015.

\section{Figure and Table Captions}


Figure 1. A user interacting with the platform

Figure 2. Details of the mobile robot

Figure 3. Extensible paddles to control the robot. From left to right: shift forward, stop, turn left, turn right

Figure 4. Messages sent between the robot's components when a movement command (cmd) is sent

Figure 5. Mindstorms ${ }^{\mathrm{TM}}$ Ev3 visual programming environment

Figure 6. Setup for the orientation task (left) and the shifting task (right)

Figure 7. Proportion of repetitions completed by subjects with no (none), mild, or severe cognitive impairment (grouped by task)

Figure 8. Time to complete a repetition of a task by subjects with no (none), mild, or severe cognitive impairment (grouped by task)

Figure 9. Number of unnecessary actions when completing a repetition of a task by subjects with no (none), mild, or severe cognitive impairment (grouped by task)

Figure 10. Shifting precision errors measured as the distance to the target when completing a repetition of a task by subjects with no (none), mild, or severe cognitive impairment (grouped by task)

Figure 11. Percentage of actions that were not successful at giving a command to the robot (grouped by level of cognitive impairment)

Figure 12. Reasons to not being able to complete a repetition of a task (grouped by task and level of cognitive impairment)

Figure 13. Example of a cognitive game to stimulate working memory with Tangibot

Figure 14. Four users playing a game to foster creativity with Tangibot

Table 1. Number (and proportion) of users with each level of cognitive impairment that expressed the specified impressions 
Table 2. Number (and proportion) of users with each level of cognitive impairment that showed the specified behaviors 